\title{
Reputación corporativa de marcas globales del sector de la cerveza: el caso Heineken
}

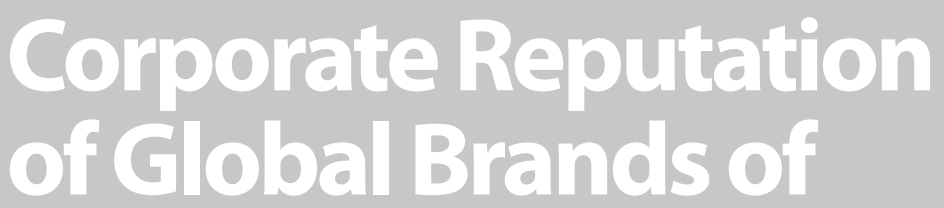
the Beer Industry: Heineken

Ana María Casado Molina

Dpto. de Economía y Administración de Empresas Universidad de Málaga acasado@uma.es

Paula Salazar de Funes Universidad de Málaga paulasalazar@uma.es
Casado Molina A. M.a , y Salazar de Funes, P., (2017)

Reputación corporativa de marcas globales del sector de la cerveza: el caso Heineken

Revista Internacional de Investigación en Comunicación aDResearch ESIC. No $15 \mathrm{Vol} 15$

Primer semestre, enero-junio 2017 · Págs. 92 a 107

DOI: 15.7263/ADRESIC.015.005 


\section{Clasificación JEL: M31, M39, L66 Palabras clave:} reputación corporativa, marca corporativa, intangible relacional, marca global, reputación de Heineken
En la industria cervecera, la tendencia del consumidor de cada país es depositar principalmente su confianza sobre las marcas de fabricación local del territorio donde habita generando así una reputación más favorable sobre estas marcas frente a otras firmas internacionales.

Frente a esta situación, el objetivo de esta investigación, es conocer frente a marcas de cerveza locales cuál es la reputación corporativa de las marcas internacionales del sector de la cerveza, concretamente, se analiza el caso de la marca Heineken en mercados locales. El estudio empírico aborda, la percepción de Heineken que existe en su ciudad de origen, Groningen, versus una ciudad no origen de otro país. Para conocer la reputación de esta marca en ambas ciudades, en primer lugar, se han estudiado, a través de fuentes secundarias, los índices y principales variables que miden la reputación corporativa; en segundo lugar, hemos realizado una encuesta adaptada al contexto cultural y socioeconómico de ambas muestras, universitarios autóctonos de su ciudad de origen y de una ciudad no origen donde está presente dicha marca corporativa.

Como resultado de la investigación, se ha demostrado que la marca corporativa global Heineken es percibida como líder no sólo en su ciudad de origen, sino también en mercados locales no origen de dicha marca. Se detecta que, su buena reputación corporativa en las distintas ciudades estudiadas viene determinada por su asociación con su marca de producto «Heineken» y no con el resto de marcas locales adquiridas por esta firma como estrategia utilizada para su posicionamiento internacional. Esta investigación pone de manifiesto que una marca de cerveza puede lograr su liderazgo internacional no sólo a través de estrategias de adquisición de marcas locales, como se ha realizado tradicionalmente en este mercado, sino a través de una estrategia de comunicación de marca global que contribuya a impulsar su reputación corporativa en los mercados donde desee operar.

\section{ABSTRACT}

\section{JEL Classification: M31, M39, L66 Key words:} corporate reputation, corporate brand, relational intangibles, global brand, Heineken's reputation
In the beer industry, the trend of consumers in each country is to trust mainly locally produced brands where they live. In this way, local brands gain a more favorable reputation than other international firms.

Taking this into account, the aim of this research is to establish the corporate reputation of international beer brands versus local brands, specifically the case of the Heineken brand in local markets is analyzed. The empirical research compares the perception of Heineken in its city of origin, Groningen, with a non-original city of another country. In order to know about the reputation of this brand in both cities, firstly, the main indexes and variables that measure the corporate reputation have been studied through secondary sources. Secondly, we carried out a survey adapted to the cultural and socio-economic context of the two samples; local university students of the city of origin and of a non-original city where this brand is present. As a result of this research, it has been shown that the global corporate brand, Heineken, is a brand leader not only in its hometown, but also in other local markets. It has been detected that its good corporate reputation in the different cities is determined by its association with its product brand «Heineken» and not with other local brands acquired by this firm as a strategy to reach its international positioning. This research shows that a brand of beer can achieve international leadership not only through local brand acquisition strategies, as has traditionally been the case in this industry, but also through a global brand communication strategy that contributes to promote its corporate reputation in the markets where this brand wants to operate. 


\section{Introducción}

Las empresas, actualmente, incorporan los activos intangibles de capital relacional tales como la reputación corporativa para conseguir una clara diferenciación y sostenibilidad en los mercados globales donde desempeñan su actividad (Fombrun y Shanley, 1990; Hur et al., 2013).

Numerosos estudios —-Thomson, MacInnis y Park, 2005; Helm, 2005; Holmes, 2000; Helm, 2007 - muestran una nítida relación directa y positiva entre la recomendación o actitud de los productos o servicios de las empresas y la reputación de su marca corporativa (Carreras, Alloza y Carreras, 2013). En esta era de la Economía de la Reputación (Losana, 2012), la marca corporativa frente a la marca de producto juega un papel muy relevante en la toma de decisión de compra por parte de los clientes (Capriotti, 2009a). La decisión de compra está cada vez más vinculada a aspectos diferenciadores de la marca corporativa en sí que de las características propias de los productos (Teresa y Blanco, 2013; Capriotti, 2009).

Ante esta situación, muchas marcas corporativas focalizan sus esfuerzos en identificar, construir y fortalecer las relaciones con sus grupos de interés o stakeholders con una mayor transparencia y confianza para diferenciarse frente a sus competidores (Compromiso RSE, 2016). Las empresas consideran que es importante apostar por la constancia en acciones corporativas coherentes basadas en valores de credibilidad y transparencia para conseguir una percepción favorable de sus stakeholders y como consecuencia, un incremento de su reputación y liderazgo en los mercados (Llorente y Cuenca, 2013; Davis et al., 2004; Wahid y Ahmed, 2011).

La internacionalización ha promovido aún más la importancia de este bien intangible. La existencia de mercados cada vez más globales y conectados hace que las empresas que operan en dichos mercados aumenten su necesidad por conseguir una clara diferenciación de su marca global ante sus competidores y así obtener una ventaja competitiva sostenible en dichos mercados.

Sin embargo, resulta interesante observar que en el comportamiento de la industria cervecera ha existido siempre una clara tendencia de confianza del consumidor hacia las marcas locales más que a las globales, es decir, aquellas fabricadas en el territorio nacional (Carceller, 2015; Cervvebel, 2016). Según el estudio Drinks International (2014) Heineken podría ser una de las marcas corporativas que podría frenar la tendencia de preferencia de las marcas locales.

En el sector cervecero, existen modelos para medir la reputación del sector y conocer como es percibido dicho sector por sus stakeholders. Entre los más relevantes se encuentran los modelos de Merco España y Reptrak (Orozco y Ferré, 2012). Sin embargo no se han encontrado estudios públicos que desarrollen un análisis comparativo de la reputación de marcas corporativas de cerveza en su mercado de origen vs otros mercados o ciudades internacionales, en el mismo periodo y para perfiles específicos de público objetivo.

En este artículo se desea aportar una nueva perspectiva sobre la reputación de marcas corporativas globales de cervezas: conocer la percepción que los stakeholders tienen sobre el comportamiento de dichas marcas en los mercados de origen (mercado local) frente a otros mercados no origen internacionales donde opera la marca durante un mismo periodo estacional. Para llevar a cabo esta investigación se abordan los siguientes pasos: en primer lugar, se realiza una revisión de los principales conceptos, índices y métricas de reputación que existen así 
como las investigaciones al respecto en el sector cervecero; en segundo lugar, se delimitan los objetivos e hipótesis de esta investigación así como la metodología a seguir; en tercer lugar, se presentan los resultados del estudio empírico; y por último, se contemplan las conclusiones principales.

\section{Revisión de la literatura}

\subsection{La Reputación Corporativa: Definición, públicos y ventajas}

La reputación de marca corporativa consiste en la percepción que los grupos de interés de una compañía tienen hacia los comportamientos y actividades que éstas desarrollan en su relación con ellos (Riel y Fombrun, 2007; Carrió, 2013). En función del grado de cumplimiento de compromisos con cada uno de sus públicos (clientes, empleados, accionistas, comunidad, etc.) las marcas adquieren una mejor o peor reputación (Wiedmann y Prauschke, 2006; Fisher-Buttinger y Vallaster, 2011).

La reputación puede estar enfocada hacia la marca de producto o la marca corporativa. La reputación de marca corporativa aborda un amplio grupo de interés frente a la de producto que se centra únicamente en el cliente (Hatch y Schult, 2010; Melo y Garrido-Morgado, 2012).
MacMillan et al. (2004) conciben el grupo de interés como aquel grupo de personas que poseen la capacidad de influir en los resultados del negocio de la organización así como en los beneficios de ésta. Existen numerosas clasificaciones de grupos de interés (Freeman, 2004; Mínguez, 2010). Tal y como se muestra en la tabla 1 , Dowling (2002) realiza dicha clasificación en función de las necesidades y valores que aportan a la empresa.

La reputación corporativa es concebida como un bien intangible de capital relacional que aporta ventajas competitivas en los mercados donde desempeña su actividad y provee de múltiples beneficios para el negocio de la firma (Casado y Peláez, 2013; Villafañe, 2004; Vergin y Dorongleh, 1998; Alloza, 2005; Monfort, 2014; Carreras et al., 2013; Frechilla y Carrillo, 2012; Rindova et al., 2005): (1) Atrae inversiones y además mejora los resultados económicos de ésta; (2) Actúa como escudo protector frente a crisis corporativas; (3 )Influye en un alto valor bursátil; (3) Fortalece el valor de la marca generando valor y crecimiento rentable del negocio; (4) Aumenta el valor comercial de los productos de los productos y servicios de la compañía, lo que supone una mayor competitividad; (5) Contribuye a la entrada a nuevos mercados; (6) Reduce la incertidum-

\section{Tabla 1 - Definición grupos de interés}

\begin{tabular}{|l|l|}
\hline Grupos normativos (reguladores) & $\begin{array}{l}\text { Dentro de este segmento encontramos a las autoridades y las agencias de evaluación } \\
\text { y certificación. Grupos con un fin común: establecer y cumplir normas. }\end{array}$ \\
\hline Grupos funcionales (trabajo) & $\begin{array}{l}\text { Compuesto por los empleados, sindicatos, suministradores y distribuidores. Su interés } \\
\text { común se basa en aportar valor y defender su estatus. }\end{array}$ \\
\hline Grupos difusos (comunidad) & $\begin{array}{l}\text { Formado por los grupos de presión, los medios de comunicación así como las distintas } \\
\text { asociaciones comunales. Su objetivo se basa en la mediación de otros segmentos. }\end{array}$ \\
\hline Clientes & Segmento más homogéneo de los cuatro. Interés común: obtener satisfacción y valor. \\
\hline
\end{tabular}


bre en las transacciones realizadas del consumidor con la empresa; (7) Aumenta la fidelización del cliente; (8) Aumenta las barreras psicológicas de entrada de otras marcas; (9) Decrecimiento de la sensibilidad del consumidor por el precio.

\section{2. Índices y métricas para medir la reputación corporativa}

Los índices de la reputación corporativa tienen el objeto de determinar niveles de reputación de una empresa (Orozco y Ferré, 2012). Existen distintas clasificaciones en la medición de reputación: monitores o herramientas de reputación; modelos holísticos, transversales o mixtos (Casado y Peláez, 2014; Toro y Pavia, 2012; Cravens, Oliver y Ramamoorti, 2003; Walker, 2010).

Carreras et al. (2013), aborda las métricas de reputación bajo una clasificación en función de dos variables contextuales: unicultural o transcultural y uni o multistakeholder.

De los distintos indices que se observan en la tabla 2, uno de los índices más relevantes y utilizados por las multinacionales a nivel global es el Reptrack Pulse (Casado, Méndiz y Peláez, 2013).
El índice Reptrack Pulse permite trabajar la reputación de las empresas globales por su característica transcultural y es aplicable a los distintos stakeholders (Ponzi, Fombrun y Gardberg, 2011). Este índice permite: diagnosticar y establecer objetivos, causas y consecuencias de la reputación de estas marcas, evaluando y estableciendo comparaciones con otras empresas del mismo sector (Hernández, Losada y Macías, 2007). (Tabla 2)

En la figura 1, se muestra cómo el modelo, sobre el que se sustenta el índice, trabaja el grado de fortaleza de la reputación existente entre la empresa y sus distintos grupos de interés, a través de diferentes indicadores: estima, admiración, impresión y confianza. Esta reputación es explicada a través de siete dimensiones que son estándar para su aplicación en los diferentes países a los que se desee aplicar: Oferta, Innovación, Trabajo, Integridad, Ciudadanía, Liderazgo y Finanzas (Ponzi, Fombrun y Gardberg, 2011).

Estas dimensiones del RepTrack se explican a través de las experiencias y percepciones que sus públicos tienen en relación a 23 atributos (véase tabla 3). Los indicadores, dimensiones y atributos que explican la reputación han sido contras-

\section{Tabla 2 • Índices de Reputación Corporativa}

\begin{tabular}{|l|l|l|l|}
\hline Índices & Modelo de RC & Transcultural/ Unicultural & Unistakeholder/ Multistakeholder \\
\hline Reptrack Pulse & Métrica de evaluaciones subjetivas & Transcultural & Multistakeholder \\
\hline Merco & Métrica objetiva & Transcultural & Multistakeholder \\
\hline CBR & Métrica de evaluaciones subjetivas & Transcultural & Unistakeholder \\
\hline Alva & Métrica objetiva & Transcultural & Multistakeholder \\
\hline Irma & Métrica de evaluaciones subjetivas & Unicultural & Multistakeholder \\
\hline Helm & Métrica de evaluaciones subjetivas & Unicultural & Unistakeholder \\
\hline
\end{tabular}




\section{Figura 1 - Dimensiones RepTrack}

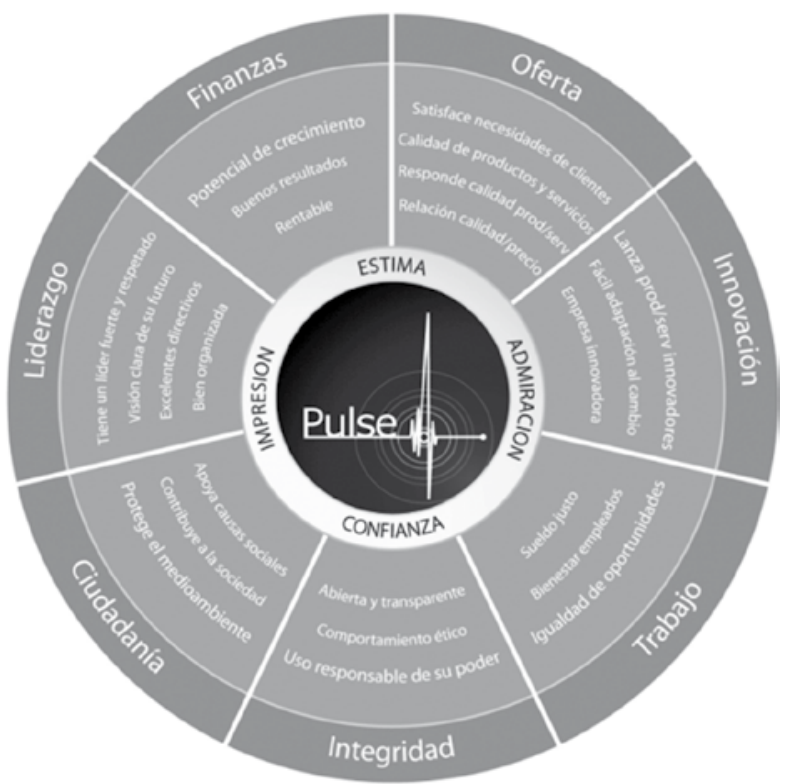

Fuente: RI, 2016

tadas y validadas empíricamente para ser aplicadas sobre cualquier tipo de público y sector empresarial (Helm, 2007; Helm 2005; Ponzi, Fombrun y Gardberg, 2011).

\section{Tabla 3 - Dimensiones de la Reputación}

\begin{tabular}{|l|l|}
\hline Dimensiones & Atributos \\
\hline Finanzas & Potencial de crecimiento, buenos resultados y rentable. \\
\hline Oferta & Satisface necesidades de clientes, calidad de productos/servicios y relación calidad/precio. \\
\hline Innovación & Lanza productos/servicios innovadores, fácil adaptación al cambio, empresa innovadora. \\
\hline Trabajo & Sueldo justo, bienestar empleados, igualdad de oportunidades. \\
\hline Integridad & Abierta y transparente, comportamiento ético, uso responsable de su poder. \\
\hline Ciudadanía & Apoya causas sociales, contribuye a la sociedad, protege el medioambiente, \\
\hline Liderazgo & Posee un líder fuerte y respetado, visión clara de su futuro y bien organizada. \\
\hline
\end{tabular}

Fuente: Ponzi, Fombrun y Gardberg, 2011 
Otro índice que trabaja la reputación transcultural es el CBR (Customer Business Reputation) o Índice de Reputación basado en el cliente. Este índice creado por Walsh y Beatty (2007) propone una evaluación transcultural unistakeholder de la reputación de marca, concretamente la reputación generada por el cliente. Dicho índice cuenta con cinco dimensiones: Orientación al cliente; Buen empleador; Empresa confiable y sólida financieramente; Calidad del producto y servicio; y, Responsabilidad social y ambiental. Tal y como se observa en la tabla 4 , cada una de estas dimensiones se describen en una serie de variables. (Tabla 4).

\subsection{La reputación de las marcas globales en el sector cervecero}

Según afirma el presidente de Cerveceros de Europa la tendencia de los consumidores de cerveza hacia marcas locales, y la búsqueda por parte de estos consumidores hacia cervezas más especiales, locales y cercanas, supone una aumento del interés de las empresas pequeñas y locales del sector a su asociación para así explotar al máximo sus fortalezas (Carceller, 2015).

Son numerosas las empresas de este sector que han llevado a cabo compras de otras marcas de cerveza para así unirse y operar de manera conjunta. La marca corporativa Heineken es una de las empresas que ha desarrollado esta estrategia. Heineken Internacional adquirió marcas de cerveza locales del país para aumentar su presencia y notoriedad de marca corporativa. Heineken Internacional opera a nivel global como marca y además cuenta con un 20\% de marcas locales para adaptarse a las culturas de los países en los que desempeña su actividad (Rivero, 2006).

Existen diversas investigaciones sobre la reputación corporativa del sector cervecero. El índice de reputación corporativa Merco (Villafañe, 2015) confirma que dentro de las empresas con mejor reputación en 2015 en nuestro país, aparecen dos firmas del sector de las cervezas: Mahou San Miguel (puesto 21) y Grupo Damm (puesto 44). Barrado (2015) estudia la reputación de la marcas de cerveza en Holanda y afir-

\section{Tabla 4 • Índice CBR}

\begin{tabular}{|l|l|}
\hline $\begin{array}{l}\text { Dimensiones } \\
\begin{array}{l}\text { Orientación } \\
\text { al cliente }\end{array}\end{array}$ & $\begin{array}{l}\text { Atributos } \\
\text { los clientes. Tratar a sus clientes de manera justa. }\end{array}$ \\
\hline $\begin{array}{l}\text { Buen empleador } \\
\text { Buena compañía para trabajar. Trato correcto. Excelente liderazgo. Gerencia con interés } \\
\text { a empleados. Buenos empleados y buena gestión. }\end{array}$ \\
\hline $\begin{array}{l}\text { Empresa confiable y } \\
\text { sólidamente financiera }\end{array}$ & $\begin{array}{l}\text { Supera a competidores. Reconoce y aprovecha oportunidades de mercado. Perspectivas de } \\
\text { crecimiento futuro. }\end{array}$ \\
\hline $\begin{array}{l}\text { Calidad del producto y } \\
\text { servicio }\end{array}$ & $\begin{array}{l}\text { Ofrece productos y servicios de calidad. Empresa sólida y confiable. Respalda los servicios } \\
\text { que ofrece. Servicios innovadores y buena relación calidadprecio }\end{array}$ \\
\hline $\begin{array}{l}\text { Responsabilidad Social } \\
\text { y ambiental }\end{array}$ & $\begin{array}{l}\text { Crea nuevos puesto de trabajo. Reduce sus ganancias para asegurar un ambiente limpio, } \\
\text { ambientalmente responsable. Apoya buenas causas. }\end{array}$ \\
\hline \begin{tabular}{l} 
Fuente: Walsh y Betty (2007) \\
\hline
\end{tabular} & \\
\hline
\end{tabular}


ma que la marca Trappe destaca por la dimensión reputacional de calidad del producto. Por último, el índice Reptrack Pulse 2015 muestra que la marca Heineken es la única firma del sector cervecero que se encuentra dentro del ranking de las TOP 100 a nivel mundial (Prado y Jonhson, 2015; Heineken, 2014).

Tras una revisión de las investigaciones existentes sobre reputación de las marcas de cerveza, no se encuentran evidencias de estudios que profundicen en el comportamiento reputacional de las marcas corporativas globales de cervezas en su país de origen vs otro país donde también desempeñen su actividad. Sin embargo, como afirma Jung y Seock (2016), resulta interesante la investigación de la reputación porque ésta es utilizada por la sociedad como diagnóstico sobre una marca y como información útil para tener una actitud o intención de conducta.

\section{Objetivos e hipótesis de la investigación}

El objetivo principal de la investigación es conocer el comportamiento de la reputación de las marcas corporativas de cerveza globales en su territorio de origen de dicha marca versus un territorio no origen de la marca y conocer si existe una mejor reputación hacia marcas locales o globales. Este estudio se centra en el caso de la marca corporativa global Heineken y en medir la reputación generada por un target específico de la misma, universitarios de la ciudad de origen holandés, Groningen, y de la ciudad española, Málaga.

Como objetivos específicos, se pretende: (1) Averiguar el conocimiento que de la marca corporativa Heineken tienen los universitarios clientes y no clientes de ésta en ambas ciudades; (2) Comparar el conocimiento que dichos universitarios tienen de la marca Heineken con res- pecto a otras marcas globales/loc ales de cerveza; (3) Conocer el sentimiento, de admiración o confianza, que le genera la marca a cada uno de estos grupos de interés (universitarios de cada ciudad); (4) Conocer qué causas (dimensiones o variables) son las que describen la reputación que cada uno de estos públicos (universitarios de esta ciudad del país de origen o no) tienen hacia la marca en el territorio de origen de la marca versus del país no origen.

Para abordar dichos objetivos y ante el vacío de investigaciones sobre el comportamiento reputacional de las marcas globales de cervezas, tal y como se abordó en el apartado del marco teórico, se plantean contrastar las siguientes hipótesis teóricas y básicas en dicha investigación:

$\mathbf{H}_{\mathrm{t} 1}$ : La mayoría de los universitarios tanto de la ciudad de origen como de la ciudad no origen conocen la marca corporativa Heineken, tanto si la consumen como si no la consumen.

$\mathbf{H}_{\mathrm{b} 1}$ : Si $\mathbf{H}_{t 1}$ es cierta, al menos el $70 \%$ de los encuestados responderá Heineken a la pregunta del cuestionario: «¿Cuál de estas empresas de cerveza conoce?».

$\mathbf{H}_{\mathrm{t} 2}$ : La mayoría de los encuestados conoce las marcas de los productos cerveceros que pertenecen a la empresa Heineken.

$\mathbf{H}_{b 2}$ : Si $\mathbf{H}_{t 2}$, es cierta al menos el $70 \%$ de los encuestados conoce las marcas que pertenecen a la empresa.

$\mathbf{H}_{\mathrm{t} 3}$ : La mayoría de los encuestados afirma que Heineken tiene una buena reputación.

$\mathbf{H}_{b 3}$ : Si $\mathbf{H}_{t 3}$ es cierta, al menos el $60 \%$ de los encuestados afirma que Heineken tiene una buena reputación.

$\mathbf{H}_{\mathrm{t} 4}$ : La mayoría de los encuestados de ambas ciudades puntúa la marca Heineken con un valor más alto en: Medio ambiente; Solidez financiera y rentable; Buena relación calidad-precio 
de sus productos; e Innovación de la empresa en sus procesos.

$\mathbf{H}_{b 4}$ : si $\mathbf{H}_{t 4}$ es cierta, al menos el $70 \%$ de los encuestados puntúa dichas variables con la calificación más alta.

$\mathbf{H}_{t 5}$ : Los encuestados de ambas ciudades (de origen y no origen) afirman que Heineken es líder en el sector de la cerveza.

$\mathbf{H}_{b 5}$ : si $\mathbf{H}_{t 5}$ es cierta, más del $50 \%$ de los encuestados afirma que la marca es líder entre las marcas de cerveza existentes en cada región.

\section{Metodología}

\subsection{Muestra}

Se realiza el estudio del comportamiento de la reputación de marcas de cervezas que actúan en su mercado de origen y en otros mercados internacionales. Se ha seleccionado la marca corporativa Heineken porque es la única marca corporativa global que lidera el Ranking de consumo de cervezas mundiales, estando en el puesto tercero (Kantar, 2015). Además, se realiza un estudio de su reputación corporativa respecto a las principales marcas corporativas con sus marcas de cerveza respectivas que operan en las dos ciudades a estudiar (ver tabla 5): su ciudad de origen, Groningen, y otra ciudad no origen, Málaga.

Según Universia España (2014) los jóvenes universitarios son un target muy habitual sensibilizado con este sector. En esta investigación se ha trabajado, precisamente, con este universo: jóvenes universitarios de entre 18 y 30 años de las dos ciudades, Ciudad de origen- Groningen- y ciudad no origen- Málaga. Para obtener ambas muestras de jóvenes se realizó sobre estos dos universos infinitos un muestreo aleatorio simple con un error muestral de $\pm 4,8 \%$. Las encuestas se aplicaron, finalmente, a dos muestras de universitarios: la primera muestra se aplicó sobre 400 estudiantes universitarios procedentes de Málaga y 400 se aplicó a universitarios procedentes de Groningen.

\subsection{Variables}

Las variables sobre las que se ha diseñado la encuesta se han extraído del modelo transcultural y multistakeholder que se aplica en el índice de reputación RepTrack, ya que la investigación persigue abordar el estudio de la reputación corporativa de una marca de cerveza global, generada por distintos grupos de interés tanto en su ciu-

\section{Tabla 5 - Marcas de cerveza en España y Holanda}

\begin{tabular}{|c|c|}
\hline España & Holanda \\
\hline $\begin{array}{l}\text { Mahou San Miguel: con } 36 \text { tipos de cervezas. Destacan: Alhambra, } \\
\text { Calsberg, San Miguel y Mahou }\end{array}$ & Babaria \\
\hline $\begin{array}{l}\text { Heineken España: } 37 \text { tipos de cerveza. Destacan: Heineken, Cruzcampo, } \\
\text { Amstel, Buckler y Guinness }\end{array}$ & Buckler(pertenece a Heineken) \\
\hline Grupo Damm: 25 tipos de cerveza. Destaca: Estrella Damm & Grolsh: Premiun blond, premiun weizen, Premiun malt, etc. \\
\hline Hijos de Rivera: 20 tipos de cervezas. Destaca: Estrella Galicia & Heineken \\
\hline Compañía cerveceros de Canarias: 12 tipos de cerveza & La Trappe:Tripel, Quadrupel, Witte Trappist, Bockbier, Isid'or \\
\hline \multirow[t]{2}{*}{ La Zaragozana: 23 tipos de cerveza } & Amstel(pertenece a Heineken) \\
\hline & Ámsterdam: Explorator, Navigator, Mariner \\
\hline uentes: Elaboración propia a partir de AA.W. (Cerveceros de España, 2015) & \\
\hline
\end{tabular}


dad de origen como en otras ciudades de países no origen donde desempeña su actividad.

La encuesta se aplica para las muestras de cada una de las ciudades, adaptando el idioma y las marcas del sector cervecero más relevante (Rivera, 2006) en cada una de las ciudades respecto a la marca corporativa global Heineken (ver tabla 5).

\section{Resultados de la investigación}

A continuación se presentan los resultados de la investigación realizada para conocer la reputación de la marca corporativa global Heineken en su territorio de origen (Groningen) versus un territorio no origen de la marca y conocer si existe una mejor reputación hacia marcas locales o hacia esta marca global por parte de los universitarios de ambos territorios.

Como se muestra en la figura 2, más del 90\% de los encuestados universitarios de ambos territorios de origen o no, conocen la marca corporativa Heineken tanto si consumen como si no consumen sus productos o marcas de cervezas (se acepta la Hipótesis 1- $\mathbf{H}_{\mathrm{t} 1}$ y $\mathbf{H}_{\mathrm{b} 1}$ ).

\section{Figura 2 - Conocimiento de la marca corporativa Heineken}

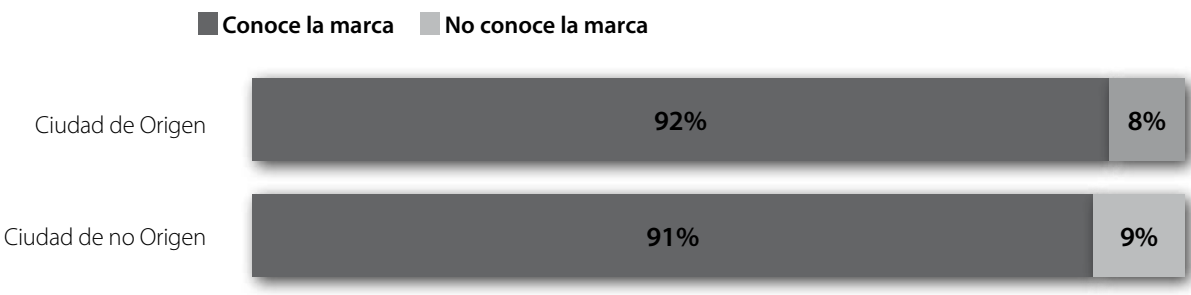

Fuente: Elaboración propia

\section{Figura 3 - Conocimiento de las marcas de cerveza pertenecientes a Heineken (Ciudad de origen)}

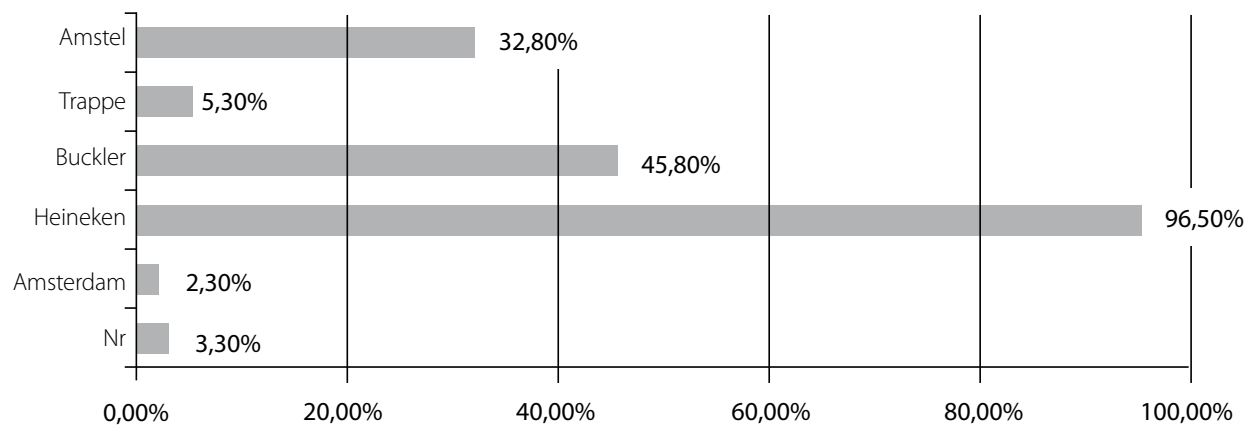


La mayoría de los encuestados de ambas muestras (ciudad de origen o no), asocian la marca de la cerveza Heineken a su marca corporativa, sin embargo en su ciudad de origen menos del 46\% reconocen el resto de marcas de cervezas pertenecientes a dicha marca global. En el caso de la ciudad no origen (Málaga) menos del 35\% la reconocen (ver figuras 3 y 4 ). Se rechaza la hipótesis $2\left(\mathbf{H}_{\mathrm{t} 2}\right.$ y $\left.\mathbf{H}_{\mathrm{b} 2}\right)$.

La empresa con mejor reputación en la ciudad de origen y no origen es la marca corporativa global Heineken, así lo consideran el 97\% de los encuestados en su ciudad de origen y el 89\% en su ciudad no origen, con una nota media por encima de los 6,3 puntos (escala Likert). Además un 63\% de los universitarios de la ciudad de origen (Groningen) confían en dicha marca cor- porativa y un 61'5\% de los encuestados de la ciudad no origen también. La hipótesis 3 de esta investigación se acepta $\left(\mathbf{H}_{\mathrm{t} 3}\right.$ y $\left.\mathbf{H}_{\mathrm{b} 3}\right)$.

Más del 70\% de los encuestados en ambas ciudades de origen y no origen puntúan a la marca Heineken con un valor más alto en las dimensiones reputacionales de Ciudadanía, concretamente en las temáticas de contribución social y medioambiente respecto a otras variables (ver figura 5). Sin embargo en el caso de los universitarios de la ciudad de origen (Groningen) la más percibida es la conciencia por la importancia del medio ambiente, sin embargo dicho atributo queda en el puesto $3^{\circ}$ para los encuestados de la ciudad no origen que relacionan más la buena relación calidad-precio de sus productos (ver tabla 6.). Por tanto, se rechaza la hipótesis $4\left(\mathbf{H}_{\mathrm{t} 4} \mathrm{y} \mathbf{H}_{\mathrm{b} 4}\right)$.

\section{Figura 4 - Conocimiento espontáneo de las marcas de cerveza pertenecientes a Heineken (Ciudad no origen)}

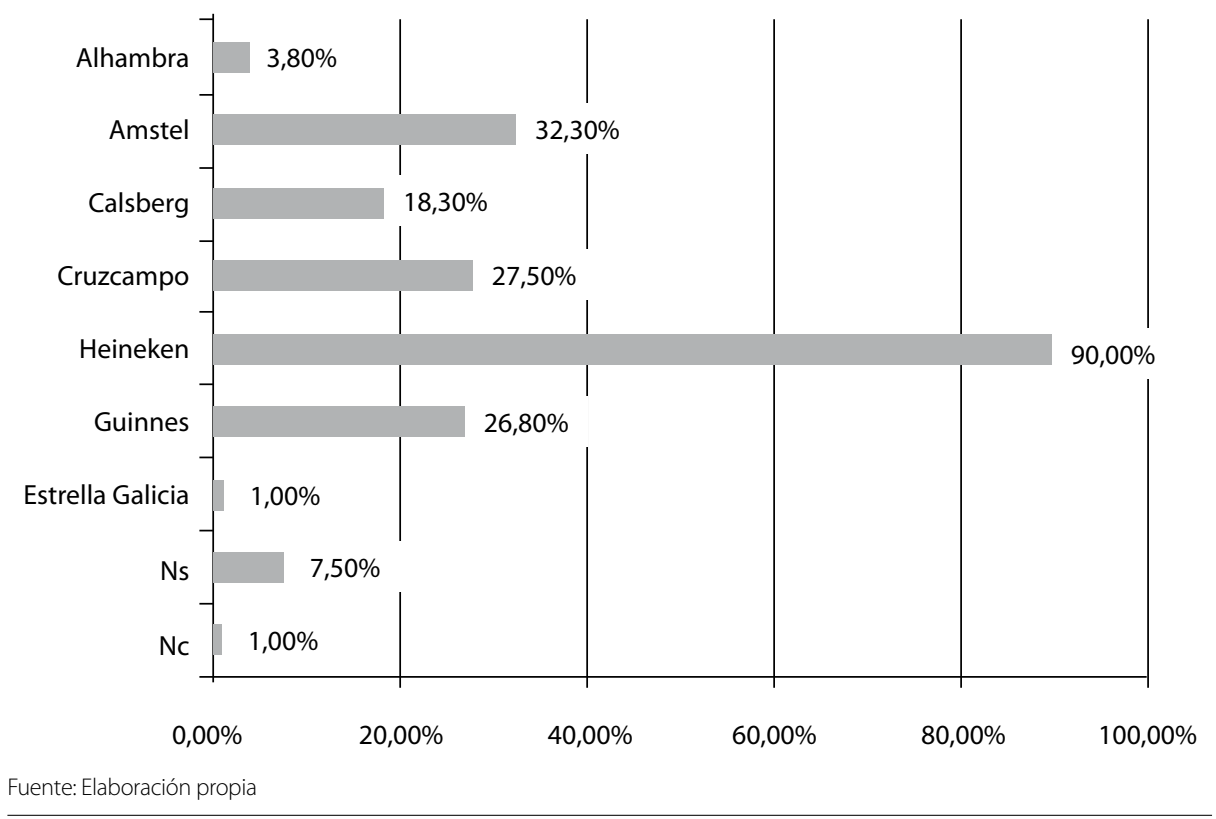




\section{Figura 5 - Percepciones de Heinieken (Temáticas Percibidas en \% de usuarios)}

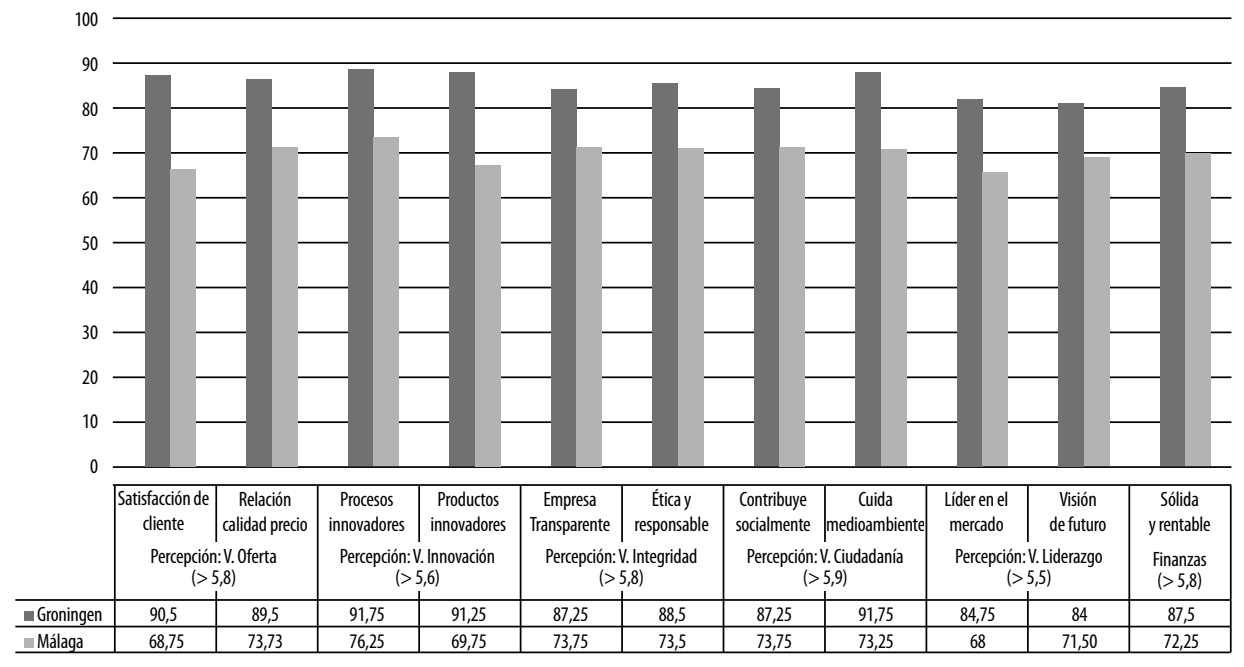

\section{Tabla $6 \cdot$ Ranking de atributos de reputación}

\begin{tabular}{|c|l|l|}
\hline Posición & Ciudad de Origen & Ciudad no Origen \\
\hline 1 & Consciencia de la importancia del medio ambiente. $\mathbf{5 . 9 8}$ & La buena relación calidad- precio de sus productos. $\mathbf{5 . 8 6}$ \\
\hline 2 & La buena relación calidad-precio de sus productos. $\mathbf{5 . 9 5}$ & La innovación de la empresa en sus procesos. $\mathbf{5 . 8}$ \\
\hline 3 & La solidez financiera y rentable. $\mathbf{5 . 9 3}$ & Consciencia de la importancia del medio ambiente. $\mathbf{5 . 8}$ \\
\hline 4 & La innovación de la empresa en sus procesos. $\mathbf{5 . 7}$ & La solidez financiera y rentable $\mathbf{5 . 8}$ \\
\hline Legenda: Escalas de valoración del O(mínimo) al 7(máximo). & \\
\hline Fuente: Elaboración propia & \\
\hline
\end{tabular}

Como se muestra en la figura 6, la marca cor- líder del mercado sin embargo sólo un 51\% de los porativa global Heineken es percibida por un $73 \%$ encuestados en la ciudad no origen consideran su de los encuestados de su ciudad de origen como liderazgo. Se acepta la hipótesis $5\left(\mathbf{H}_{\mathrm{t} 5} \mathrm{y} \mathbf{H}_{\mathrm{b} 5}\right)$. 


\section{Figura 6 - Marcas corporativas líderes en el sector de la cerveza en Ciudad de Origen}
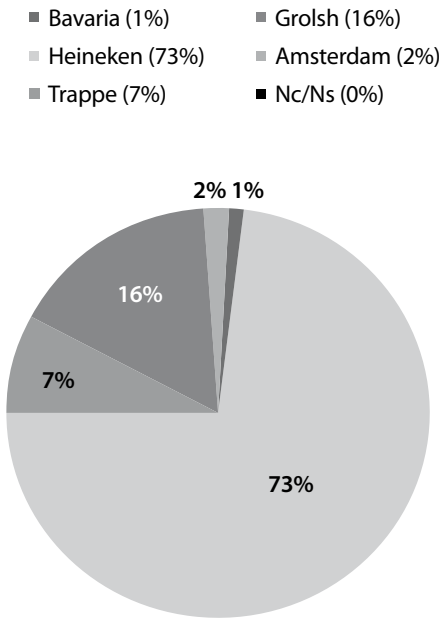

Marcas corporativas líderes en el sector de la cerveza en Ciudad no Origen

- Mahou San Miguel (38\%) - Compañía cerveceros

- Heineken (51\%) de Canarias (1\%)

- Grupo Damm (7\%)

- Hijos de Rivera (3\%)

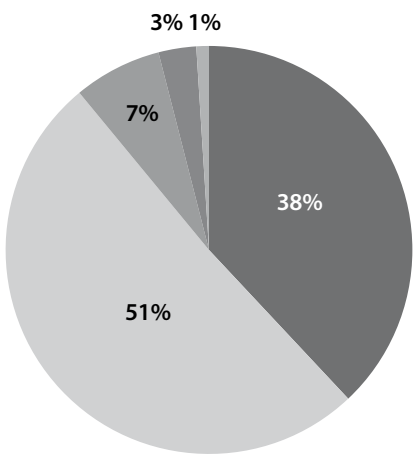

Los resultados obtenidos de las distintas temáticas analizadas por parte de los perfiles analizados - universitarios de la ciudad de origen o no- han sido los siguientes:

\section{Conocimiento de la marca Heineken y sus} productos. Los universitarios de la ciudad de origen y no origen tienen un alto conocimiento de la marca corporativa Heineken. Sin embargo en la ciudad de origen (Groningen) tienen un mayor conocimiento de las marcas de productos de Heineken corporación (42\%) frente a los encuestados de la ciudad no origen (menos del 35\%).

\section{Reputación de la marca corporativa Heine-} ken. En la ciudad de origen, el 97\% de los encuestados consideran que tiene una buena reputación frente al 89\% de la ciudad no origen. La percep- ción positiva se genera en ambas ciudades, tanto por parte de consumidores de marca como no consumidores, ya que en ambas muestras analizadas, el porcentaje de consumidores habituales de productos que así la perciben es superior al 50\% (59,2\% en el caso de la ciudad de origen y $58,3 \%$ en la ciudad no origen de la marca corporativa).

\section{Temáticas relacionadas con la percepción} o reputación de Heineken. Para los encuestados de la ciudad de origen, el atributo al que más asocian la reputación de Heineken es la innovación en los procesos de dicha marca y en la temática de ciudadanía, medioambiente principalmente. Sin embargo para los universitarios de la ciudad no origen es además de la innovación en los procesos, la relación calidad-precio de sus 
productos, la transparencia de la marca con su entorno y su contribución social.

\subsection{Limitaciones de la investigación}

En esta investigación se ha abordado los perfiles de stakeholders más afines del sector cervecero, concretamente de la marca Heineken. En próximos estudios se abordará el resto de stakeholders.

\section{Conclusiones}

En este artículo se ha analizado la reputación corporativa de una marca global de cerveza como es Heineken frente a sus marcas competidoras en su ciudad de origen versus una ciudad no origen donde también se comercializan sus productos. Los universitarios clientes y no clientes de ambas ciudades conocen la marca corporativa y la consideran mayoritariamente líder del mercado de cerveza de su país, a pesar de no ser una cerveza de ámbito local. Sin embargo es interesante resaltar que en ambas ciudades la segunda cerveza que ha sido percibida como líder en cada uno de los mercados es de fabricación local. En esta investigación, se concluye que la marca corporativa Heineken, es una excepción en el comportamiento de los posicionamientos de las marcas de cerveza a nivel mundial. Esta marca es la única marca que sin ser una marca de fabricación local, tiene una reputación global de cerveza que tras- pasa el posicionamiento de liderazgo del sector cervecero en cada país ya que hasta ahora era propio de las marcas de fabricación local.

En esta investigación se ha mostrado que existe un leve conocimiento sobre los productos de cerveza o marcas de cerveza que comercializa esta marca corporativa en las distintas ciudades, tanto de origen como no origen. La reputación de una marca corporativa global en el sector cervecero, como Heineken, no proviene de la percepción de sus marcas de cervezas de carácter local, sino por la asociación, por parte de los encuestados, de las experiencias principalmente de la marca de producto «Heineken» con su marca corporativa.

Las marcas corporativas globales de cerveza, como es el caso de Heineken, pueden tener un comportamiento reputacional positivo muy similar tanto en su país de origen como en un país no origen. No siempre estas percepciones favorables en los diferentes países- de origen o noson lideradas por marcas exclusivamente de fabricación local de cervezas. Además dichas percepciones favorables sobre las marcas globales de cerveza no son siempre de consumidores sino también proceden de un público potencial de consumo que tienen una actitud favorable hacia la marca corporativa y escasa experiencia con las marcas de producto de dicha firma. 


\section{Bibliografía}

Alloza, A. (2005). La reputación corporativa, la eficacia de la comunicación, la marca y la creación de valor. Investigación y Marketing,_86, 6.

Barrado, J.A. (2015, 15 de marzo). Cerveza Trapense. Cerveza independiente. Disponible en: https://cerveceraindependiente.com/abadias-trapenses.

Capriotti, P. (2009). Branding corporativo. Fundamentos para la gestión estratégica de la identidad Corporativa. Chile: Colección Libros de la Empresa.

Capriotti, P. (2009a). De la imagen a la reputación: análisis de similitudes y diferencias. Razón y palabra, 70 (3), 1-10.

Carceller, D. (2015). La posible adquisición de SABMiller por parte de Anheuser Busch-InBev y sus consecuencias en el mercado de la cerveza. Madrid: Universidad Pontificia de Comillas. Carreras E., Alloza A. y Carreras A. (2013). Reputación corporativa. Madrid: LID Editorial empresarial.

Carrió, M. (2013). Gestión de la reputación corporativa: convierte lo que piensan y dicen de ti en un mejor activo. Barcelona: Llorenç Rubió.

Casado, A.M., Méndiz, A. y Peláez, J.I. (2013). La evolución del DirCom: de gestor de la comunicación a estratega de la reputación. Comunicación y sociedad, 26(1), 47-69.

Casado, A.M., y Peláez, J.A. (2013). Un modelo de desarrollo profesional para la mejora de la reputación corporativa. Questiones publicitarias: Revista internacional de comunicación y publicidad, 18, 75-92.

Casado, A.M. y Peláez, J.I. (2014). Intangible management monitors and tools: reviews. Expert Systems with Applications, 41(4), 1509-1529.

Cervvebel. (2016, 17 de marzo). Historia de la cerveza: su descubrimiento. Disponible en: http://www.cervebel.es/cerveza_descubrimiento.htm.

Cerveceros de España (2015, 1 de abril). Informe socioeconómico del sector de la cerveza. Disponible en: http://www. cerveceros.org/pdf/CE_Informe_socioeconomico_2015.pdf.

Compromiso RSE (2016, 17 de marzo). Responsabilidad social. Disponible en: http://www.compromisorse.com/responsabilidad-social/alimentacion-bebidas-hogar-e-higiene/ mahou-san-miguel.

Cravens, K., Oliver, E.G., y Ramamoorti, S. (2003). The Reputation Index: Measuring and Managing Corporate Reputation. European Management Journal, 21(2), 201-212.
Davies, G., Chun, R., da Silva, R. V., y Roper, S. (2004). A corporate character scale to assess employee and customer views of organization reputation. Corporate Reputation Review, 7(2), 125-146.

Dowling, G. (2000). Creating Corporate Reputations: Identity, Image and Performance: Identity, Image and Performance. Oxford: OUP.

Drinks International. (2014, 21 de marzo). World's 50 Best Bars Brands Report: Beer. Disponible en: http://www.drinksint.com/news/fullstory.php/aid/4281/Worlds50BestBars_ Brands_Report:_Beer.htm

Llorente y Cuenca (2013). Reputación y Liderazgo. Madrid: Anatomía de red.

Fisher-Buttinger, C., y Vallaster, C. (2011). Corporate branding and corporate reputation: Divided by a shared purpose? En Helm, S., Liehr-Gobbers, K., \& Storck, C. (Eds.). (2011 Reputation Management (pp. 59-73). Berlin: Springer. Frechilla, M., y Carrillo, M. (2012). La reputación corporati$v a=$ beneficios. Madrid: Grupo Reputación Corporativa

Freeman, R.E.(2004). Strategic Management: A stakeholder approach. Boston: Pitman.

Fombrun, C., y Shanley, M. (1990). What's in a name? Reputation building and corporate strategy. Academy of Management Journal, 33(2), 233-258.

Hatch, M.J., y Schultz, M. (2010). Esencia de marca (Vol. 1). Madrid: LID Editorial.

Heineken. (2014, 1 de abril). Heineken, informe de sostenibilidad 2014. Disponible en: http://www.heinekenespana.es/ HK_Espana_Informe_Sostenibilidad_2014.pdf._

Helm, S. (2005). Designing a formative measure for corporate reputation. Corporate Reputation Review, 8(2), 95-109.

Helm, S. (2007). The role of corporate reputation in determining investor satisfaction and loyalty. Corporate Reputation Review, 10(1), 22-37.

Helm, S., Liehr-Gobbers, K., \& Storck, C. (Eds.). (2011). Reputation management. Springer Science $\&$ Business Media.

Hernández, M., Losada, A., y Macías, A. (2007). Estrategia y conducta social de la organización. Salamanca: Universidad Pontificia Salamanca.

Holmes, J.G. Social Relationships: The Nature and Function of Relational Schemas. European Journal of Social Psychology, 30(2000), 447-495. 
Hur, W. M., Kim, H., y Woo, J. (2013). How CSR leads to corporate brand equity: Mediating mechanisms of corporate brand credibility and reputation. Journal of Business Ethics, 125(1), 75-86.

Jung, N. Y., y Seock, Y. K. (2016). The impact of corporate reputation on brand attitude and purchase intention. Fashion and Textils, 3(1), 20-45.

Losana, Á. A. (2012). Reflexiones sobre la reputación necesaria. adComunica, 3, 27-47.

MacMillan, K., Money, K., Downing, S., y Hillenbrand, C. (2004). Giving your organisation SPIRIT: an overview and call to action for directors on issues of corporate governance, corporate reputation and corporate responsibility. Journal of General Management, 30(2), 15-42.

Melo, T., y Garrido-Morgado, A. (2012). Corporate reputation: A combination of social responsibility and industry. Corporate Social Responsibility and Environmental Management, 19(1), 11-31.

Mínguez, M.I. (2010). Los públicos en las relaciones públicas. Barcelona: Editorial UOC.

Monfort, A. (2014, 10 de marzo). Reputación corporativa: ¿Cómo beneficia a la empresa? Disponible en: http://abelmonfort.com/reputacion-corporativa-beneficios-empresa.

Orozco, J., y Ferré Pavia, C. (2012). El ADN de la marca. La concepción de sus valores intangibles en un contexto dialogado. Signo \& Pensamiento, 31(61), 56-71.

Pintado, T. y Sánchez, J. (coords) (2013). Imagen Corporativa: Influencia en la gestión empresarial (2a.ed.). Madrid: ESIC Editorial.

Ponzi, L.J., Fombrun, C.J., y Gardberg, N.A. (2011). RepTrak ${ }^{\mathrm{TM}}$ pulse: Conceptualizing and validating a short-form measure of corporate reputation. Corporate Reputation Review, 14(1), 15-35.

Prado, F. y Johnson E. (2015). Reptrack Pulse 2015. Madrid: RI. Reputation Institute. (2016, 30 de marzo.) What is my company's reputation? Disponible en: http://www.reputationinstitute.es

Riel, C.B. van, y Fombrun, C.J. (2007). Essentials of corporate communication: Implementing practices for effective reputation management. New York: Routledge.
Rindova, V. P., Williamson, I. O., Petkova, A. P., y Sever, J. M. (2005). Being good or being known: An empirical examination of the dimensions, antecedents, and consequences of organizational reputation. Academy of Management Journal, 48(6), 1033-1049.

Rivero, A.F. (2006). Enciclopedia de los alimentos: Cervezas. Distribución y consumo, 16(87), 107-118.

Thomson, M., MacInnis, D.J., y Park, C. W. (2005). The ties that bind: Measuring the strength of consumers' emotional attachments to brands. Journal of consumer psychology, 15(1) 77-91.

Toro, J. A. O., y Pavia, C. F. (2012). Los índices de reputación corporativa y su aplicación en las empresas de comunicación. En Comunicació i risc: III Congrés Internacional Associació Espanyola d'Investigació de la Comunicación (p. 42) Tarragona: Universitat Rovira i Virgili.

Universia España (2014). ¿Qué tipo de vida llevan los universitarios? Universia España.

Vergin, R.C., y Qoronfleh, M.W. (1998). Corporate reputation and the stock market. Business Horizons, 41(1), 19-26.

Villafañe, J. (2004). La buena reputación. Madrid: Ed. Pirámide.

Villafañe \& Asociados. (2015). Merco 2015. Madrid: Villafañe \& Asociados.

Wahid, N. A., y Ahmed, M. (2011). The effect of attitude toward advertisement on Yemeni female consumers' attitude toward brand and purchase intention. Global Business and Management Research, 3(1), 21-29.

Walker, K. (2010). A systematic review of the corporate reputation literature: Definition, measurement, and theory. Corporate Reputation Review, 12(4), 357-387.

Walsh, G., y Beatty, S. E. (2007). Customer-based corporate reputation of a service firm: scale development and validation. Journal of the Academy of Marketing Science, 35(1), 127 143.

Wiedmann, K.P. y Prauschke, C. (2006, mayo 25-28). How do stakeholder alignment concepts influence corporate reputation? The role of corporate communication in reputation building. Ponencia presentada en el 10th RI Conference on Reputation, Image \& Competitiveness, Nueva York, EE.UU. 